\title{
Influenza B Antigen Measurement
}

National Cancer Institute

\section{Source}

National Cancer Institute. Influenza B Antigen Measurement. NCI Thesaurus. Code C120679.

The determination of the amount of the Influenza B antigen in a sample. 